\title{
EVALUACION DEL DAÑO CAUSADO POR Diatraea saccharalis Fabricius EN EL CULTIVO DE MAIZ (Zea mays L.) EN EL MEDIO SINU
}

\author{
DAMAGE EVALUATION OF Diatraea saccharalis Fabricius \\ O N MAIZE (Zea mays L.) PLANTS IN THE MID SINU VALLEY
}

\author{
Gloria E. Serna ${ }^{1}$; José F. Garcés ${ }^{1}$; Jorge E. M ejía ${ }^{2}$ y Claudio R. Fernández ${ }^{2}$
}

\begin{abstract}
RESU MEN
El siguiente estudio se realizó en el primer semestre del 2003 en los predios de la Universidad de Córdoba, Montería, Córdoba a una altura de 14 m.s.n.m con humedad relativa de $80 \%, 28{ }^{\circ} \mathrm{C}$ de temperatura promedio mensual y $1200 \mathrm{~mm}$ de precipitación. Los maíces estudiados fueron los híbridos SV-1127, C-343, HR-661 y la variedad ICA V-156. Se utilizó un diseño experimental de bloques completamente al azar con cuatro repeticiones. La unidad experimental fue una parcela de 5 surcos por $10 \mathrm{~m}$ de largo, para un área de $40 \mathrm{~m}^{2}$. Se evaluó el porcentaje de infestación (número de plantas con orificios de salida de adultos de D. saccharalis) y el porcentaje de intensidad de infestación (número de entrenudos afectados por tallo, total de entrenudos dañados, número de perforaciones de salida de adultos y la ubicación en el tallo) e impacto en el rendimiento. El tratamiento con mayor porcentaje de infestación natural fue el híbrido C-343 con $11.75 \%$. El tratamiento que presento mayor porcentaje de intensidad de infestación natural fue el HR-661 con 9,625\%; aunque esta variable no presentó diferencia significativa entre los tratamientos. Los híbridos no presentaron diferencia significativa en producción con relación a los de la zona. La altura de planta y la altura de mazorca no presentaron diferencia significativa entre los tratamientos. Los híbridos evaluados no presentaron tolerancia al ataque del Diatrea saccharalis.
\end{abstract}

Palabras claves: Barrenador, Infestación, intensidad de infestación, rendimiento.

\begin{abstract}
The following study was carried in the first semester of 2003 in the University of Córdoba, (Montería, Córdoba), with 14 m.a.s.l. $80 \%$ relative humidity, $28{ }^{\circ} \mathrm{C}$ temperature and $1200 \mathrm{~mm}$ year rainfall. The genotypes were hybrid SV 1127, C 343, HR 661 and ICA V 156 variety. A complete block randomized design was used with four treatments and four replicates. The experimental unit was a 5 plant row $(10 \mathrm{~m}$ long) for an area of $40 \mathrm{~m}^{2}$. The evaluated variables were natural infestation percentage, intensity of
\end{abstract}

\footnotetext{
IIngeniero Agrónomo. Asistente Técnico Particular.

${ }^{2}$ Ingeniero Agrónomo. M.Sc. Facultad de Ciencias Agrícolas, U niversidad de Córdoba, Grupo de Investigación en Cultivos Tropicales de Clima Cálido. E-mail: jomequin@hotmail.com; cfernandezher@hotmail.com
} 
damage level and impact of the damage on genotype yield. Hybrid C 343 showed the highest level of natural infestation (11.8\%), although no significant differences were observed among the genotypes. H ybrid HR 661 presented the highest intensity damage (9,6\%); however, no significant differences among genotypes were detected. The evaluated genotypes did not show significant differences with respect to yield, plant height, and ear height. No genotype showed tolerance against Diatraea saccharalis attack.

Key words: Borer, infestation, intensity of infestation, yield.

\section{INTRO DUCCIO N}

El maíz es uno de los cultivos de gran importancia económica en el departamento de Córdoba con un área de siembra durante el primer semestre de 2005 de 33310 ha en maíz tecnificado y 18000 ha en maíz tradicional, los cuales generaron 1'128.820 jornales aproximadamente. Entre los limitantes fitosanitarios de este cultivo se destaca el barrenador del tallo Diatraea saccharalis, el cual ocasiona un daño inicial a las hojas y al realizar su primera muda larval perfora el tallo de la planta destruyendo la médula en forma descendente, ocasionando el acame de las plantas y por consiguente disminuyendo la producción (FEN ALCE, 2005).

Este insecto es considerado una de las plagas de mayor importancia en el cultivo de maíz, ya que la frecuencia e intensidad del ataque ha aumentado en los últimos años debido al incremento del área, condiciones ambientales favorables, uso inapropiado de insecticidas e ineficientes prácticas culturales (Altieri, 1976).

El daño ocasionado por D. saccharalis es variable con la edad o tamaño de la planta, puesto que en las más jóvenes daña los entrenudos basales, mientras que en plantas de mayor edad afecta del tercer al séptimo entrenudo (Cevallos, 1970). El debilitamiento del tallo ocasionado porD. saccharalis, hace que los maíces de porte alto en el departamento de Córdoba sean susceptibles al volcamiento al poner menos resistencia al viento. De no evitar este daño la producción se disminuye del 25 al 30\%, siendo el nivel crítico el $10 \%$ de plantas con larvas de primer instar (Lobatón, 1973).

Los monitoreos del barrenador del tallo de maíz en el departamento de Córdoba realizados por Villarreal y Rojano (2000), evidencian el incremento en la incidencia del insecto en los maíces híbridos 4004, M aster y C-343 en ocho fincas sumando un área de 200 ha. Los híbridos de maíz evaluados entre los 108 a los 123 días de sembrados, presentaron desde el 3.7 al $100 \%$ de perforaciones promedio por planta que oscilaron entre 0.3 a $11.6 \%$. Igualmente se determinó que los cultivos más cercanos a las ciénagas presentaron ataques tempranos atribuidos a la presencia de pastos naturales y cultivos de maíz sembrados bajo la modalidad tradicional que sirvieron de reservorio a la plaga; dichos ataques incidieron en la reducción de mazorcas entre el 8 al 12\%, además del incremento en \$25 $\mathrm{kg}^{-1}$ para la recolección manual del maíz en el suelo.

Villarreal y Rojano (2002), registraron incidencias del barrenador del tallo con valores desde 23.8 al $100 \%$, un promedio de perforaciones por planta del 0.3 al $11.6 \%$ y el 1.8 al $91 \%$ de plantas volcadas en lotes sembrados con maíces híbridos M aster, 4004, C-343 y HR-661. Igualmente, Villarreal (2003), registró en el municipio de Montería, larvas de Diatraea en mazorcas, siendo uno de los factores que incidió en la presencia en un $59 \%$ de patógenos como Penicillium sp. 
El monitoreo periódico del daño del barrenador del tallo de maíz, ofrece información actualizada de la tolerancia de los híbridos al ataque por el insecto, es por ello que la presente investigación permitió determinar el daño ocasionado por el insecto, su porcentaje de infestación y el efecto en la producción en los maíces SV-1127, C-343, HR-661, ICA V-156.

\section{MATERIALES Y METODOS}

El siguiente estudio se realizó en el primer semestre del 2003 en predios de la Universidad de Córdoba, ubicada en el municipio de Montería a $8^{\circ} 44^{\prime}$ de latitud Norte y $75^{\circ} 53^{\prime}$ de longitud Oeste con respecto al meridiano de Greenwich, a una altura de 14 m.s.n.m., humedad relativa de $80 \%$, temperatura promedio mensual de $28^{\circ} \mathrm{C}$, precipitación promedio anual de 1200 $\mathrm{mm}$ y brillo solar promedio de 6.8 horas diarias. Ecológicamente la zona corresponde según la clasificación de Holdrige a la denominación de bosque seco tropical (Bst) y de acuerdo a la formación agroecológica se clasifica como Cj lo cual indica que los suelos son de incipiente desarrollo (Entisoles e inceptisoles).

El experimento se estableció bajo condiciones de campo en un diseño experimental de bloques completamente al azar con cuatro tratamientos (híbridos SV1127, C-343, HR- 661 y la variedad ICA V156) y cuatro repeticiones. La unidad experimental fue una parcela de 5 surcos por $10 \mathrm{~m}$ de largo, para un área de $40 \mathrm{~m}^{2}$, con distancia entre surcos de $0.8 \mathrm{~m}$ y $0.16 \mathrm{~m}$ entre plantas. Las variables evaluadas fueron: porcentaje de infestación ha ${ }^{-1}$ por Diatraea saccharalis (número de plantas con perforaciones de salida del adulto o sea número de plantas afectadas); porcentaje de intensidad de infestación (número de entrenudos afectados por tallo, total de entrenudos dañados, número de perforaciones de salida de adultos, la ubicación en el tallo) y efecto en el rendimiento.

La evaluación del daño porD. saccharalis se realizó en la época de cosecha, se tomaron 100 plantas al azar por parcela de los surcos centrales de izquierda a derecha, las plantas recolectadas se retiraron del lote, se deshojaron y se retiraron las mazorca. A todas las plantas se le cuantificaron los entrenudos por tallo, tomando como primer entrenudo el localizado a nivel de suelo, ya que en éste se ubica el ataque del barrenador. Posteriormente se separaron los tallos sanos de los que presentaron perforaciones de salidas del insecto. Se registró la ubicación de las perforaciones con respecto al tallo y el número de entrenudos dañados y sanos; igualmente, se realizaron cortes longitudinales en todos los tallos afectados, se tomaron registros fenológicos cada ocho días, tomando al azar cuatro plantas dentro de cada parcela, ello con el propósito de determinar el posible efecto del ataque del Diatraea sobre las variables de altura, floración y rendimiento.

\section{RESULTADOS Y DISCUSION}

\section{Porcentaje de infestación natural (PIN)}

El tratamiento que tuvo un mayor porcentaje de infestación fue el C-343, con $11.75 \%$ de infestación, seguido de los tratamientos SV1127 con $11.25 \%$, el ICA V-156 con $10.25 \%$ y el HR-661 con $5.5 \%$ de infestación. Estos resultados estadísticamente no presentan diferencia significativas entre tratamientos (Figura 1).

Estos resultados difieren de los encontrados por Rincón y Tisnes (1982) en maíces evaluados en el Valle del Cauca con infestaciones entre 15 y $73 \%$. Estos porcentajes de infestación altos posiblemente se deben a que en el Valle del CaucaDiatraea saccharalis es una plaga endémica debido a que el cultivo de la caña de azúcar es su huésped principal. 


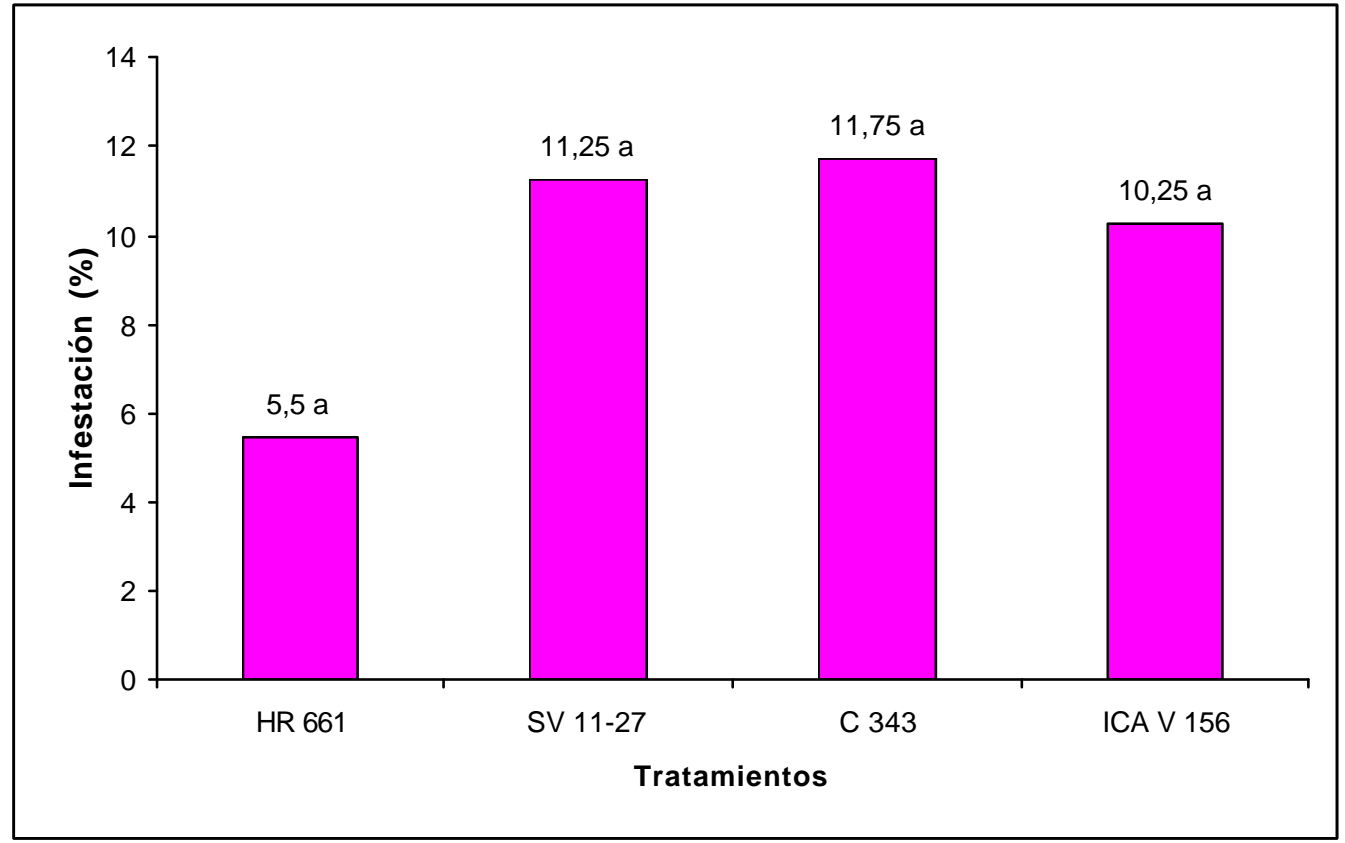

Figura 1. Porcentaje de infestación de Diatraea saccharalis en los maíces híbridos C-343, SV-1127, HR-661 y la variedad ICA V-156.

\section{Porcentaje de intensidad de infestación natural (PII).}

El tratamiento con mayor porcentaje de intensidad de infestación natural fue el HR-
661 con $9.63 \%$, seguido de los tratamientos SV-1127 con $8.95 \%$, C-343 con $8.53 \%$ y el ICA V-156 con $7.88 \%$. Esta variable estadísticamente no presentó diferencia significativa entre los tratamientos (Figura 2).

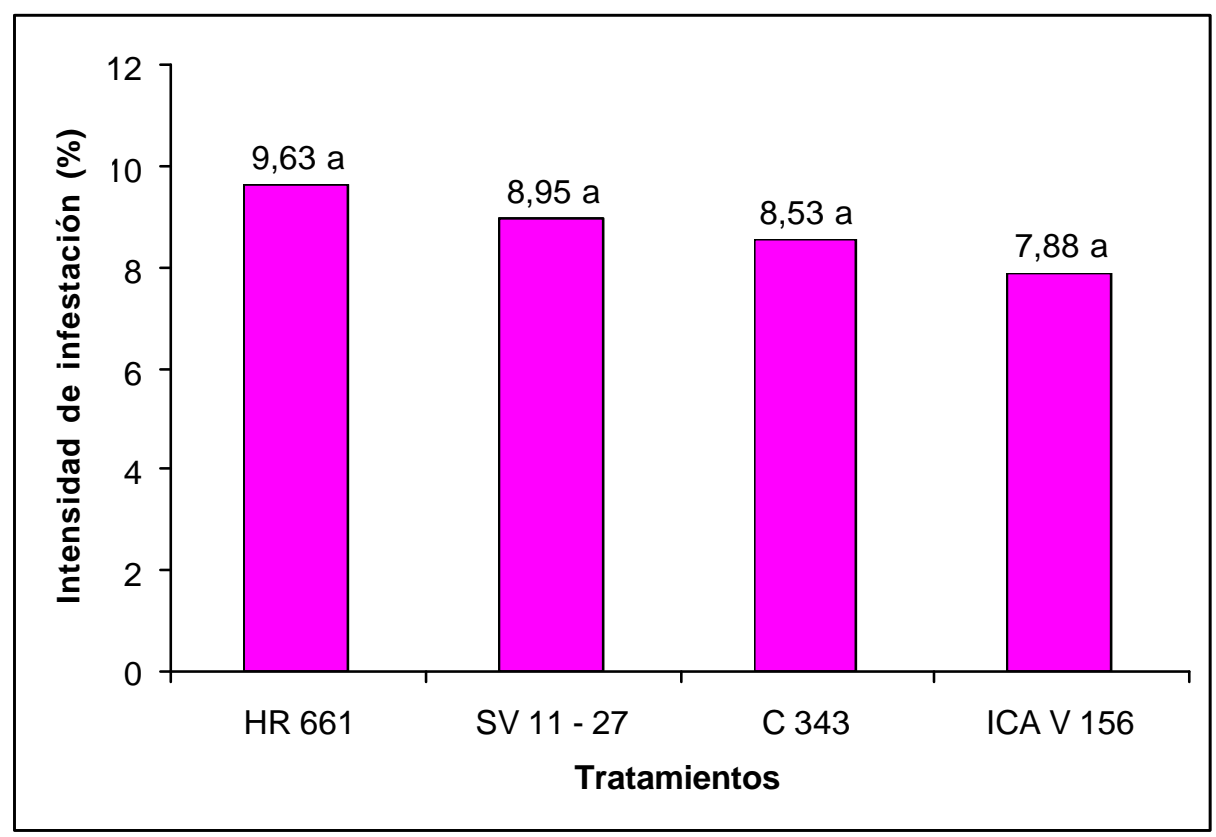

Figura 2. Porcentaje de intensidad de infestación de Diatraea saccharalis en los maíces híbridos C-343, SV-1127, HR-661 y la variedad ICA V-156. 


\section{Ubicación de las perforaciones de salida de $D$ iatraea saccharalis}

Los datos de porcentaje de orificios de salida por entrenudo (Tabla 1), muestran que para el híbrido HR-661 el $93.94 \%$ de éstos se concentran en los primeros nueve entrenudos, siendo el 2, 6 y 8 los de más altos porcentajes. En el genotipo SV-1127 el $88.33 \%$, los orificios de salida se concentraron en los primeros seis entrenudos, siendo el 2, 5 y 6 los más afectados. En el material C-343 el $86.24 \%$ de los orificios de salida se presentaron en los primeros seis entrenudos, siendo el 1, 2 y 3 los de más altos porcentajes, mientras que en la variedad ICA V-156 el $89.9 \%$ de los orificios se concentraron en los primeros siete entrenudos, siendo 4, 5 y 6 los más afectados.

Estos resultados son similares a los encontrados por M uñoz (1994), Arango et al. (1985), quienes registraron que más del $90 \%$ de los orificios de salida causados por D. saccharalis se concentran en los primeros ocho entrenudos, por tanto, evaluando éstos se puede estimar con alta aproximación el daño total. Sin emabrgo, estos resultados difieren de los reportados por Caicedo y O campo (1983) quienes encontraron un promedio de entrenudos dañados de 0.25 2.45 y 2.75 entre el segundo y el séptimo entrenudo, respectivamente. De otro Iado, Muñoz (1994) registró que en los entrenudos 2,3 y 4 se concentra el 41 al $50 \%$ del total de los orificios de salida de $D$. saccharalis y que el daño en las plantas grandes se ubicó entre el tercer y séptimo entrenudo, pero en general sin considerar tamaños, el tercer entrenudo fue el más atacado. El mismo estudio afirma que en la medida que aumenta la edad de la planta el daño causado por la larva se va acumulando en los entrenudos inferiores.

Tabla 1. Porcentaje de orificios de salida y ubicación de las perforaciones de salida de Diatrea saccharalis en genotipos de maíz.

\begin{tabular}{ccccc}
\hline $\begin{array}{c}\text { Entrenudos } \\
\text { evaluados }\end{array}$ & H R-661 & SV-1127 & C-343 & ICA V-156 \\
\hline 1 & 12.12 & 10 & 13.8 & 7 \\
2 & 18.18 & 30 & 29.3 & 7 \\
3 & 0 & 11.67 & 13.8 & 11.6 \\
4 & 12.12 & 8.33 & 8.62 & 18.6 \\
5 & 0 & 13.33 & 12.1 & 16.6 \\
6 & 15.15 & 15 & 8.62 & 20.1 \\
7 & 12.12 & 3.33 & 3.45 & 9.3 \\
8 & 15.15 & 1.67 & 3.45 & 2.3 \\
9 & 9.1 & 6.67 & 3.45 & 4.65 \\
10 & 6.06 & 0 & 1.72 & 2.3 \\
11 & 0 & 0 & 1.72 & 0 \\
12 & 0 & 0 & 0 & 0 \\
13 & 0 & 0 & 0 & 0 \\
14 & 0 & 0 & 0 & 0 \\
15 & 0 & 0 & 0 & 0 \\
\hline
\end{tabular}




\section{Altura de mazorca}

El genotipo con mayor altura de mazorca fue el HR-661 con $126.75 \mathrm{~cm}$, seguido por SV$1127 \mathrm{con} 123 \mathrm{~cm}$, el C-343 con $118.5 \mathrm{~cm}$ y el ICA V-156 con $113.75 \mathrm{~cm}$ de altura. Esta variable estadísticamente no presentó diferencia significativa entre tratamientos (Figura 3).

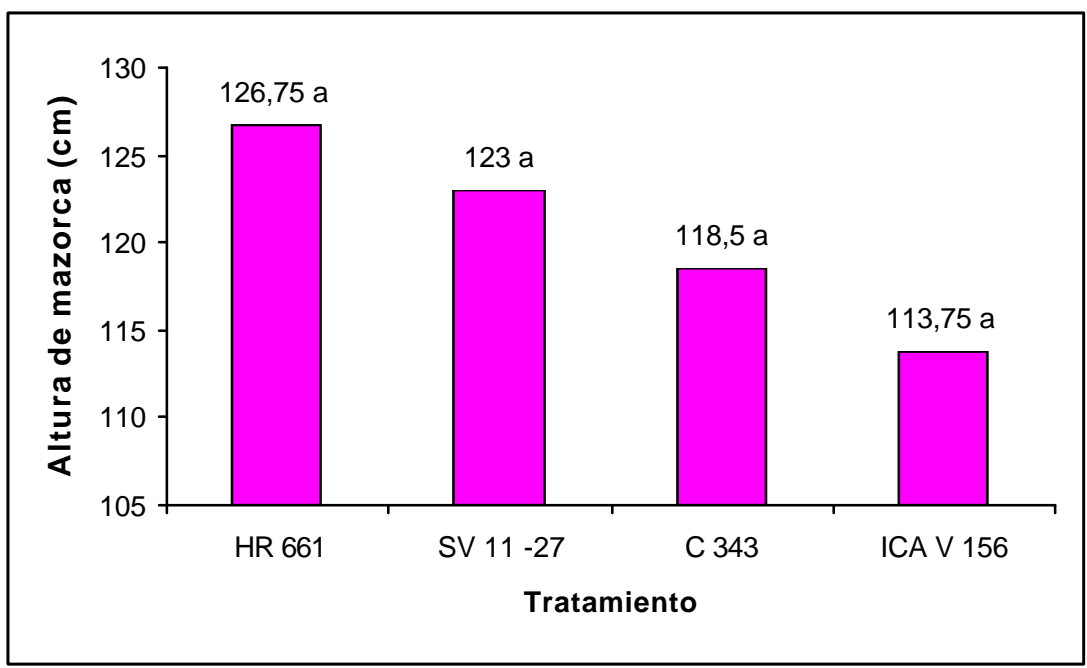

Figura 3. Altura de mazorca en las plantas de los diferentes materiales en el estudio para evaluar daño de D. saccharalis.

\section{Altura de planta}

El genotipo que presentó el mayor crecimiento fue el ICA V-156 con una altura de $233.63 \mathrm{~cm}$, seguido por el híbrido C-343 con $229.81 \mathrm{~cm}$, el SV-1127 con $213.75 \mathrm{~cm}$ y el HR-661 con $205.56 \mathrm{~cm}$. Para esta variable no se presentó diferencia significativa entre los tratamientos (Figura 4).

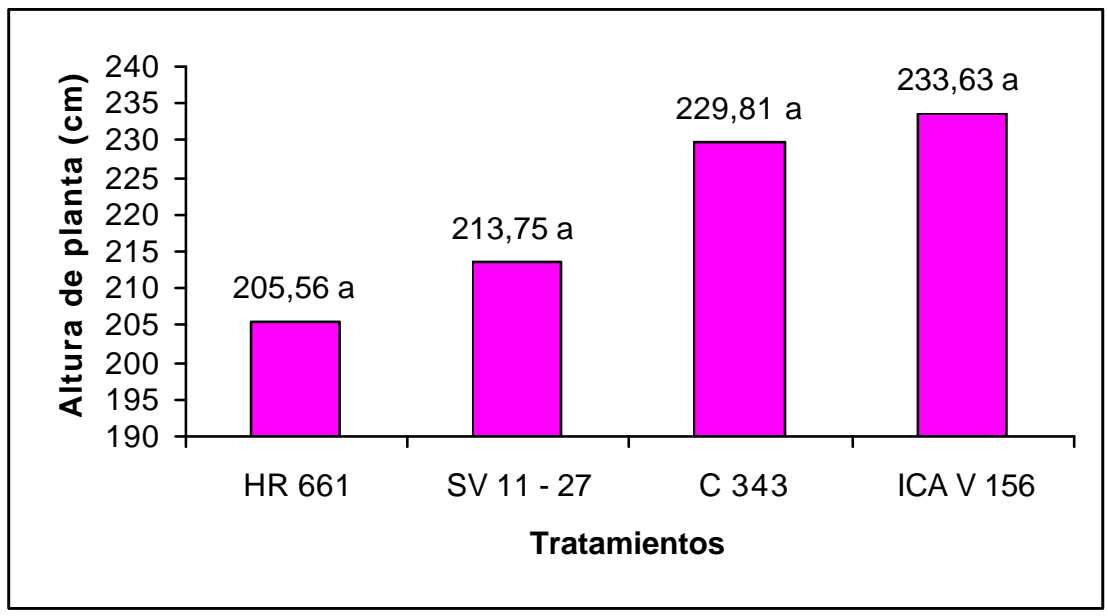

Figura 4. Altura de planta en los diferentes tratamientos en estudio. 


\section{Rendimiento}

El mayor rendimiento se presentó en el híbrido SV-1127 con $6970.8 \mathrm{~kg} \mathrm{ha}^{-1}$, seguido de los tratamientos ICA V-156 con $6775.8 \mathrm{~kg}$ $\mathrm{ha}^{-1}$, el C-343 con $5896.3 \mathrm{~kg} \mathrm{ha}^{-1}$ y el HR661 con $4820.4 \mathrm{~kg} \mathrm{ha}^{-1}$. Para esta variable no hubo diferencia significativa entre los tratamientos (Figura 5).

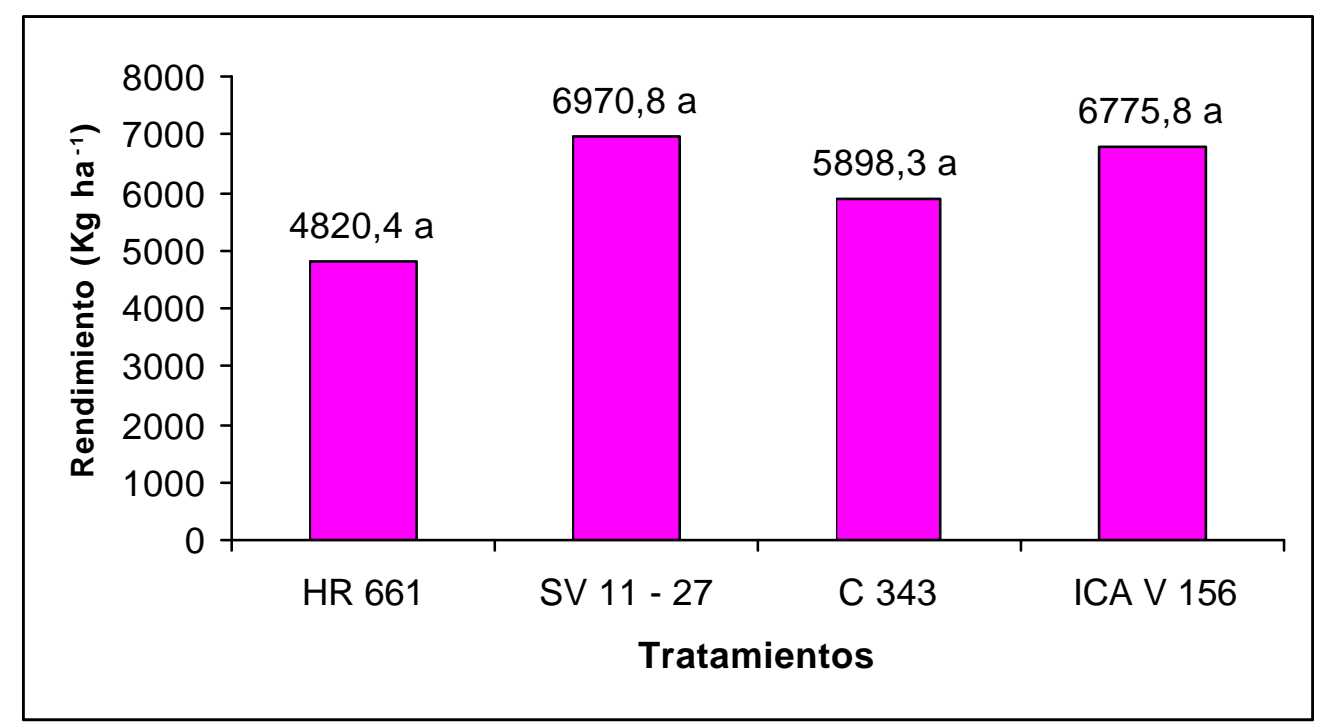

Figura 5. Rendimiento en $\mathrm{kg} \mathrm{ha}^{-1}$ de los diferentes tratamientos en estudio.

\section{CONCLUSIONES}

- Los híbridos y la variedad no presentaron tolerancia al ataque del Diatraea saccharalis bajo las condiciones evaluadas.

- El ataque del Diatreae saccharalis se localiza desde el primer entrenudo hasta el décimo, concentrado en un $90 \%$ en los primeros seis entrenudos.

- Con respecto al rendimiento asociado al daño producido por el Diatraea saccharalis, no se presentaron diferencias significativas entre los híbridos y la variedad en la zona del Sinú Medio.

\section{BIBLIO G RAFIA}

Altieri, M. 1976. Regulación ecológica de plagas en agrosistemas tropicales en mono y policultivos de maíz y fríjol, diversificados con malezas. Programa para estudios graduados, Universidad Nacional - Instituto Colombiano Agropecuario, Bogotá, $150 p$
Arango, R.; Lemos, G. y Morales, C. 1985. Evaluación del daño de Diatraea spp.(Lepidoptera : Pyralidae) y su efecto en el rendimiento de genotipos de maíz y sorgo (Sorghum bicolor) en el Valle del Cauca. Universidad Nacional de Colombia, Palmira. 
Caicedo, A. y O campo, R. 1983. Evaluación del daño causado por Diatraea spp. en los cultivos de maíz y sorgo en los municipios localizados en el departamento del Valle. Tesis Ingeniero Agrónomo, Universidad Nacional de Colombia, Palmira

FENALCE. 2005. El Cultivo de maíz en Córdoba. Evaluación de áreas de siembra. Boletín de información, Fenalce, Bogotá, p.4

Lobatón, V. 1973. Informes de visita técnica a cultivos de maíz (MonteríaCórdoba). Documento de trabajo. Instituto Colombiano Agropecuario (ICA), Cereté

Muñoz, J. 1994. Evaluación del Diatraea spp. y sus efectos en la producción de grano y semilla de trece genotipos de maíz. Universidad Nacional de Colombia, Palmira
Rincón, A. y Tisnes, G. 1982. Evaluación del daño de Diatraea spp. en los cultivos de maíz y sorgo en cuatro zonas en el departamento del Valle-Colombia. Tesis Ingeniero Agrónomo, Universidad Nacional

Villarreal, N . 2003. Informe de visita técnica en cultivos de Maíz en las Palomas (M ontería-Córdoba). D ocumento de trabajo, Instituto Colombiano Agropecuario (ICA), $4 p$.

Villarreal, N. y Rojano, E. 2000. Informe de visita de inspección a cultivos de maíz en Córdoba. Documento de trabajo, Instituto Colombiano Agropecuario (ICA). Cereté-Córdoba.

Villarreal, N. y Rojano, E. 2002. Manejo Integrado de Plagas en maíz. Centro Diagnóstico Vegetal (CISA). CeretéCórdoba. 